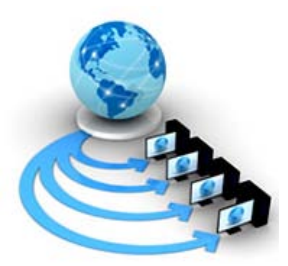

RESEARCH PAPER

\author{
Available Online at www.ijarcs.info
}

\title{
MODIFIED WEINER FILTER FOR IMPROVING QUALITY OF MRI IMAGE FOR DETECTION OF DISEASES
}

\author{
Rama Saini \\ Mtech Scholar \\ SSIET,Dinanagar
}

\author{
Priya \\ Assistant Professor \\ SSIET,Dinanagar
}

\begin{abstract}
MRI image capturing process is such that they are prone to unidentified noise. MRI image is used to diagnose a disease. Clarity is utmost necessity in such environment. Noises which can corrupt the image involve salt and pepper, Gaussian, shot noise etc. detection and removal of such noise from within the image is critical. Improving image quality is aim of this paper. Buffer is implemented to remove overlapped pixels and then wiener filter is implemented to smoothen the image. The simulation is conducted in MATLAB and noise is introduced using point spread function (PSF). Noise introduction is the first step after loading MRI image. Buffer mechanism implements uniqueness and size is reduced considerably. Weiner filter application is last step in the modified approach leading to smoothness in image. Obtained results prove the worth of study.
\end{abstract}

Keywords: MRI Image, Salt and Pepper, Gaussian, Buffer, Weiner filter, PSF(Point Spread Function)

\section{INTRODUCTION}

MRI imaging requires clarity since they are used in diagnosis of diseases. [1]introduces time dependent magnetic resonance imaging. Signal to interference plus noise ratio(SINR) is calculated to prove the validity of research. [2]proposes breast cancer detection technique. Fuzzy c means clustering is used for the same. Wisconsin Prognostic Breast Cancer (WPBC) Data Sets is used to analyse the disease. [3] proposed two methods that eliminate the overlapping in the image by blocking certain projection rays by the use of additional shielding between the pinhole plate and the detector. Two techniques to achieve this are proposed. First, the approximate filter involving contrast to noise ratio is used. Second, different amount of overlapping is considered for checking the accuracy of the filter. In the [4] proposes technique to remove noise from digital images of ancient or old documents, identification of occultation area from the old image is recognised and removed. Anomaly is identified from both side of the pixel. Hence the approach is twofold. [5]proposes the description of Weiner filtering for smoothening of images. The wiener filter introduces high degree of accuracy by reducing the mean square error from within the image.

In the existing papers overlapping of pixels is handled by the use of complex strategies. The strategies are expensive in nature. In order to overcome the problem we propose a new algorithm which will reduce the overlapping pixels using threshold and wiener filtering for efficient restoration process. In this method pixel positions are stored within the buffer. The new positions are compared with the existing positions if they are same then they will be eliminated from the result. The previous work is focused on the enhancement without considering the complexity and cost associated with the system. The proposed work concentrate on the reducing the complexity and improve performance by using buffer method. The proposed work is organised as follows

a. Obtain the MRI image from the image set. The image set is gathered from the internet source. b. Introduce noise within the image for experimentation. The noise is introduced by the use of PSF.

c. Apply Buffer mechanism to store the pixel positions iteratively. This process introduces uniqueness and removes overlapping if any.

d. Apply Weiner filter on a obtained image from step c to smoothen the image.

e. Compare results against the normal Weiner filter to prove worth of the study.

\subsection{Dataset Description}

Dataset used is derived from the internet. The MRI images are obtained from the internet and stored within the folder. The dataset is given as

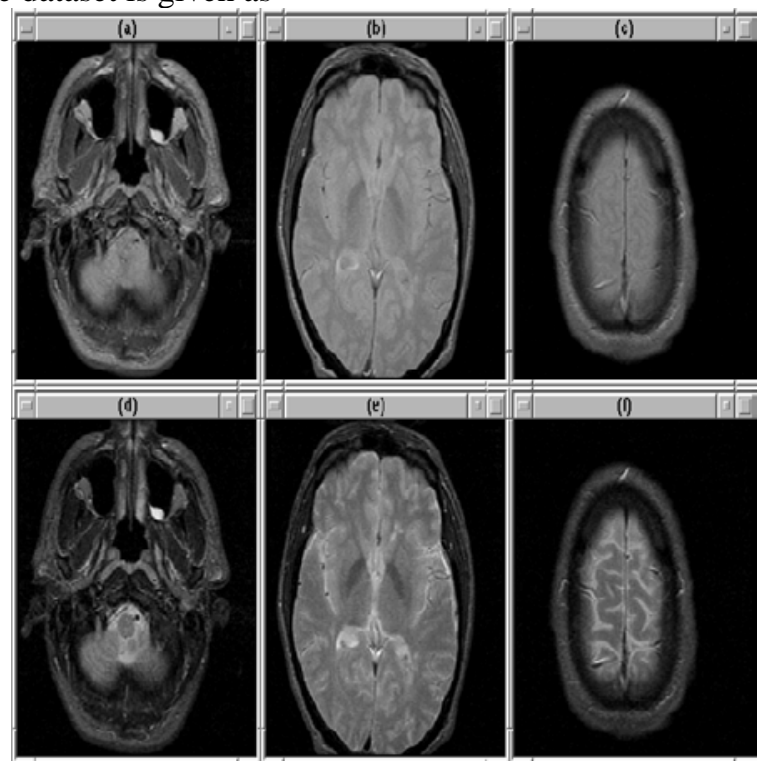

Figure 1: MRI dataset derived from the internet. Image set derived from the internet if described as under 
Table 1: Description of image set

\begin{tabular}{|l|l|l|l|}
\hline Image Set & Description & Size & Type \\
\hline Image1 & .jpeg & $320 \times 240$ & Black and White \\
\hline Image 2 & .jpeg & $320 \times 240$ & Black and White \\
\hline Image 3 & .jpeg & $320 \times 240$ & Black and White \\
\hline Image 4 & .jpeg & $320 \times 240$ & Black and White \\
\hline Image 5 & .jpeg & $320 \times 240$ & Black and White \\
\hline
\end{tabular}

The images obtained from the internet are resized to $320 x 240$. The resizing is critical to introduce uniformity.

Rest of the paper is organised as-section 2 provides related work, section 3 provides details of proposed work, section 4 provides performance analysis, section 5 provides conclusion and future work and last section give reference.

\section{RELATED WORK}

Existing literature works towards handling noise from the image. Most common noise present within the images is salt and pepper noise.

\subsection{Salt And Pepper Noise Handling Mechanism}

[6]propose two phase mechanism to handle salt and pepper noise. The first phase is used to handle noise by the use of adaptive median filter and second phase is used to restore the image using specialized regularization method. In the salt and pepper noise model observe gray scale level associated with gray scale image is listed as

$$
y_{i_{j}}=\left\{\begin{array}{c}
S_{\min } \text { wit } \square \text { probabilty } p \\
S_{\max } \text { wit } \square \text { probability } q \\
x_{i j} \text { wit } \square \text { probabilty } 1-p-q
\end{array}\right.
$$

The term $r$ is introduced for defining noise level represented as $\mathrm{r}=\mathrm{p}+\mathrm{q}$. To handle such as noise a window is defined denoted with $\mathrm{W}$. Window size is adjusted to allow or block certain pixels which becomes corrupted. [7] analyze different types of median filters used to handles impulse noise also known as salt and pepper noise.[8]-[10] Adaptive median filter is one such technique used to handle salt and pepper noise. Window is defined to handle such noise. Switching median filter is another such technique used to handle salt and pepper noise. Switching median filter uses following technique to handle such noise

$$
r_{i j}=\min \left\{\left|X_{i j} \times k_{p}\right| p=1,2,3,4\right\}
$$

Value of $r_{i j}$ is large when corrupted by noise and is small when signal is fair or is unaffected. This value is used to determine noise within the image.

\subsection{Blur Removal Techniques}

Blur commonly occurred within the images due to linear motion. Most common technique to restore the image is by the use of Weiner filter. The captured image in case of motion is corrupted as pixels are distorted due to colour patterns given through the following equation.

$$
G(u, v)=F(u, v) H(u, v)
$$

$\mathrm{F}$ is a Fourier transformation of ideal image. $\mathrm{H}$ is a sine function. In case, $\mathrm{G}$ and $\mathrm{H}$ are known then by the use of inverse filtering original image can be restored. [11]proposes input-output relation of an image for the detection of anomaly within the image.

$$
x(m)=\sum_{k=0}^{p-1} w_{k} y(m-k)
$$

In the above equation $\mathrm{x}(\mathrm{m})$ are the discrete time signals $\mathrm{W}$ is the Weiner filter coefficient. The obtained pixel intensity value is compared with the original intensity in order to obtain the error rate denoted with $\mathrm{e}(\mathrm{m})$.

$$
e(m)=x(m)=W^{t} Y
$$

The value of error rate gives magnitude of blurriness and implementation of filtering mechanism in order to remove the same. [12]suggest the application of Weiner filter. The application is widely used for the estimation of stationery signals. The error rate is observed in this case to determine the accuracy of Weiner filter.

\subsection{Contrast Enhancement}

[13], [14]Contrast enhancement is generally accomplished by the use of histogram equalization technique. [13]explore the disadvantage of histogram equalization and propose a technique to enhance contrast without losing brightness. The proposed $\mathrm{HE}$ is known as multi histogram equalization technique. it is assumed that $\mathrm{H}(\mathrm{x})$ is the histogram of the image and $\mathrm{N}$ is the total number of pixel within the image. The probability density function is evaluated as

$$
P(x)=\frac{n_{k}}{N}
$$

Where $\mathrm{n}_{\mathrm{k}}$ is the histogram generated at $\mathrm{k}=1,2,3,---, \mathrm{N}$, Where $\mathrm{N}$ is the total number of pixel. Cumulative frequency distribution is obtained after obtaining $\mathrm{P}$ as

$$
C(k)=\sum P(j)
$$

Histogram equalization can be computed from $\mathrm{C}(\mathrm{k})$ as

$$
T[i]=\left\{\frac{G-1}{a * b}\right\} * c[i]
$$

$\mathrm{G}$ indicates the Number of gray scale pixels present, $\mathrm{a}$ and $\mathrm{b}$ indicates the number of rows and columns present within the image.

\section{PROPOSED WORK}

The proposed work is used to improve the MRI images for better understanding of diseases detection. The proposed work uses the resources from the internet and nearby health care centers. The dataset describes the images which are used to resolve the problems present within them. The steps associated with the proposed approach are described in this section. 


\subsection{Pre-Processing}

The image is loaded from the image set and noise is introduced by the use of PSF (Point Spread Function). This step is critical in order to check the validity of technique proposed. The obtained image after noise introduction is displayed. Original image is also retained so that output generated from modified wiener filter can be compared against the original image. Result is produced in terms of accuracy.
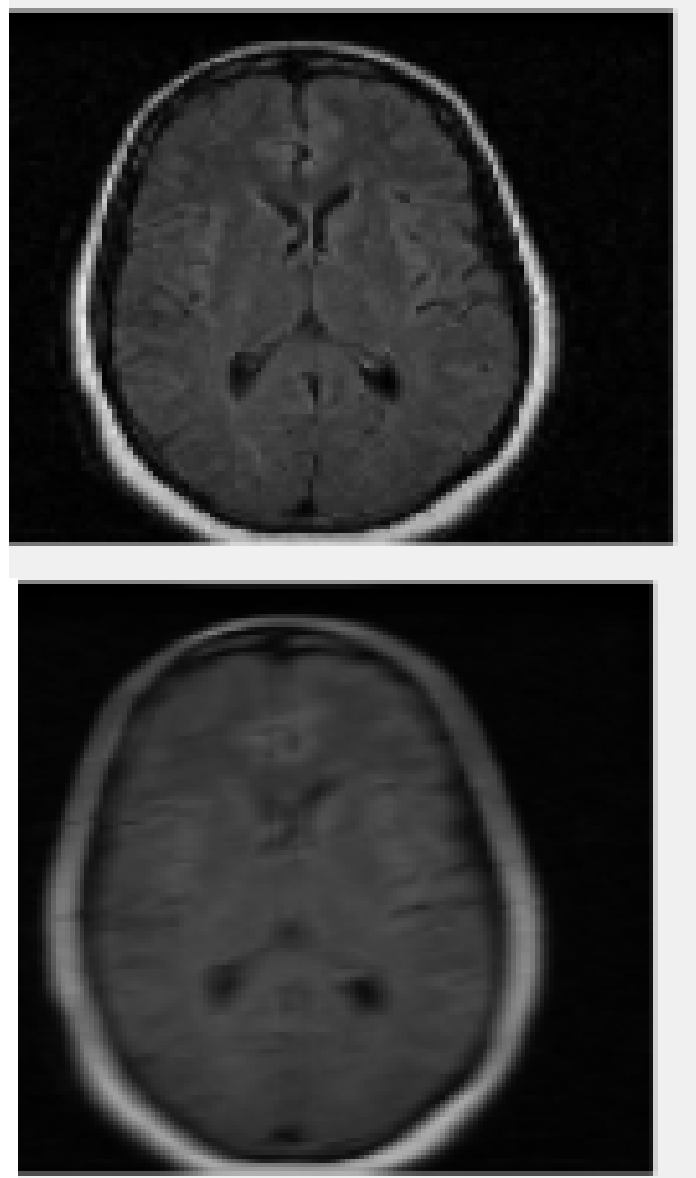

Figure 2: First image shows original MRI image and second image shows image after noise introduction

Noise is introduced with variables p and q. The motion blur is introduced by the use of additional parameter $\mathrm{r}=\mathrm{p}+\mathrm{q}$. Inequalities used are shown through equations as

$$
y_{i_{j}}=\left\{\begin{array}{c}
S_{\min } \text { wit } \square \text { probabilty } p \\
S_{\max } \text { wit } \square \text { probability } q \\
x_{i j} \text { wit } \square \text { probabilty } 1-p-q
\end{array}\right.
$$

\subsection{Eliminate Redundant Pixels}

The redundant pixels represent overlapping. Overlapped pixels reduce clarity within the image. Size is also increased considerably as redundancy is present within the image. Redundancy tackling mechanism is employed in proposed work to eliminate overlapping of pixels.

$$
\text { Buffer }_{i}=\left\{\begin{array}{l}
1 \text { if Pixel already present } \\
0 \text { if pixel is not repeated }
\end{array}\right.
$$

If pixel is not already present then it is stored within the buffer for plotting. If pixel is already plotted then its further plotting is prohibited. In other words only unique pixels are plotted by the use of this mechanism. After the application of this overlapping elimination mechanism image plots are shown as

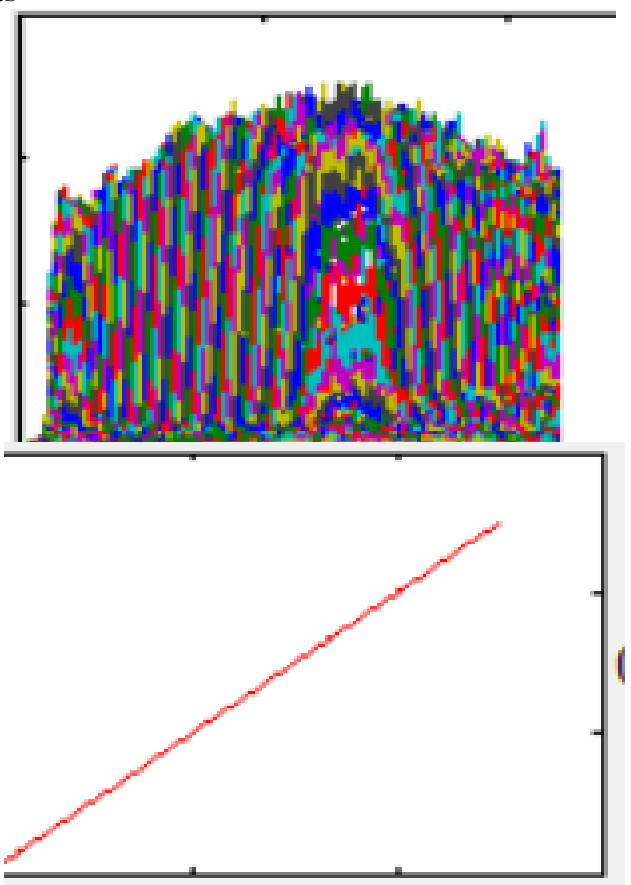

Figure 3: First image show image density without Buffer mechanism and second image show image density with buffer mechanism.

Linearity in image indicates more clarity and lower size as compared to non linear images.

\subsection{Weiner Filtering For Image Storation}

After image overlapping is reduced, image is required to be restored. The restoration mechanism used in this work is Weiner Filtering. The wiener filtering mechanism [15] as discussed in detail is implemented in this section using the equations

$$
G(u, v)=\frac{H(u, v) P(u, v)}{|H(u, v)|^{2} * P(u, v)+P(u, v)}
$$

Partitioning of image is done to reduce the complexity of image. Partitioning is represented with $\mathrm{P} . \mathrm{H}(\mathrm{u}, \mathrm{v})$ is the degradation function. Most of the noise from the signal is removed and image is restored. The signal to noise ratio is observed in this case.

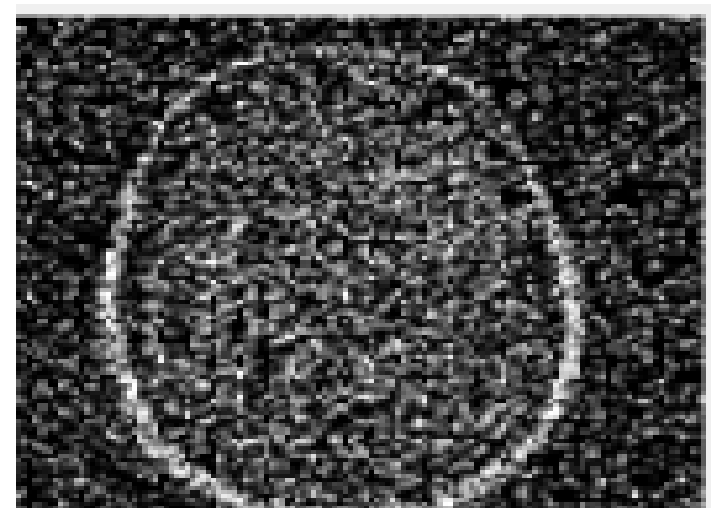




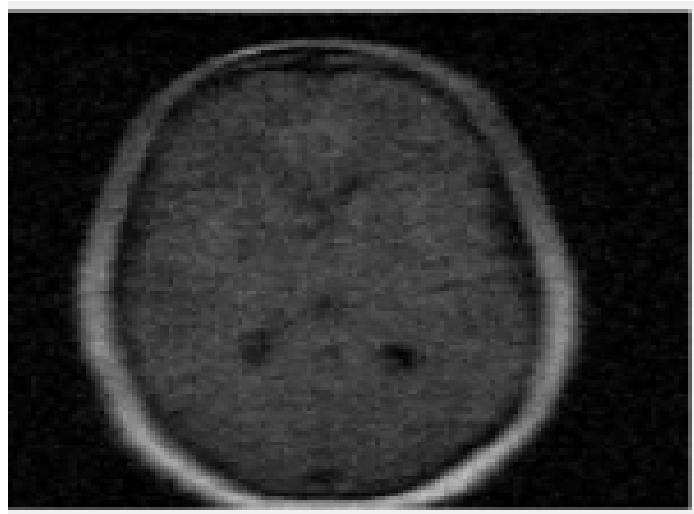

Figure 4: First image shows the restored image after first attempt and second image shows restored image after second attempt.

Weiner filtering is applied multiple times to image obtained after buffer mechanism in order to obtain desired level of accuracy.

\subsection{Proposed Flowchart}

The methodology to be followed in the proposed system is listed in terms of flowchart in this section.

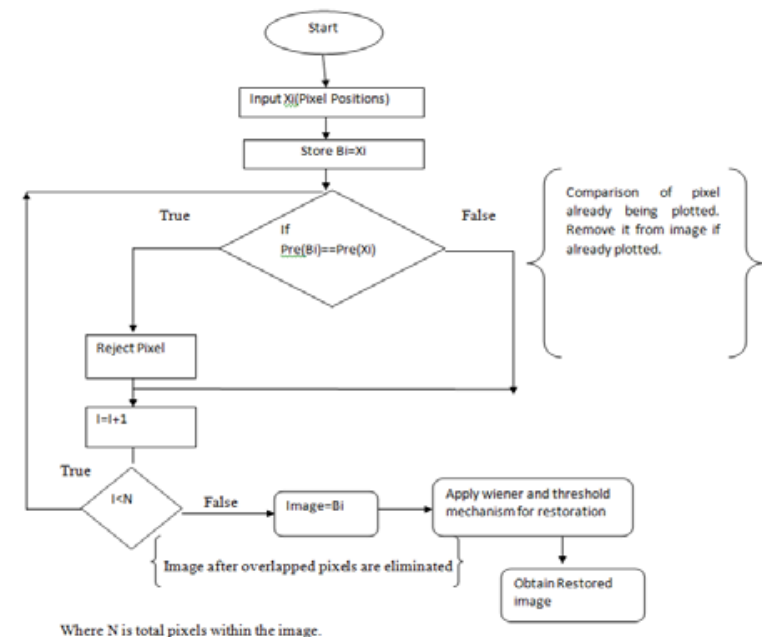

Figure 5: Flowchart of Proposed System

\section{PERFORMANCE COMPARISON}

The proposed system generates parameters such as CPU_Time, F-score and Tanimoto distance. The proposed technique gives better result than normal wiener filtering mechanism in terms of listed parameters.

Table 2: Result Comparison in terms of various parameters

\begin{tabular}{|l|l|l|l|}
\hline \multirow{2}{*}{ Image } & Parameter & Weiner Filter & $\begin{array}{l}\text { Modified } \\
\text { Filter }\end{array}$ \\
\hline \multirow{2}{*}{ Image1 } & CPU Time & 5.5365 & 2.5658 \\
\cline { 2 - 4 } & F-Score & 0.756 & 0.9234 \\
\hline & Tanimoto Distance & 0.9635 & 0.0826 \\
\hline
\end{tabular}

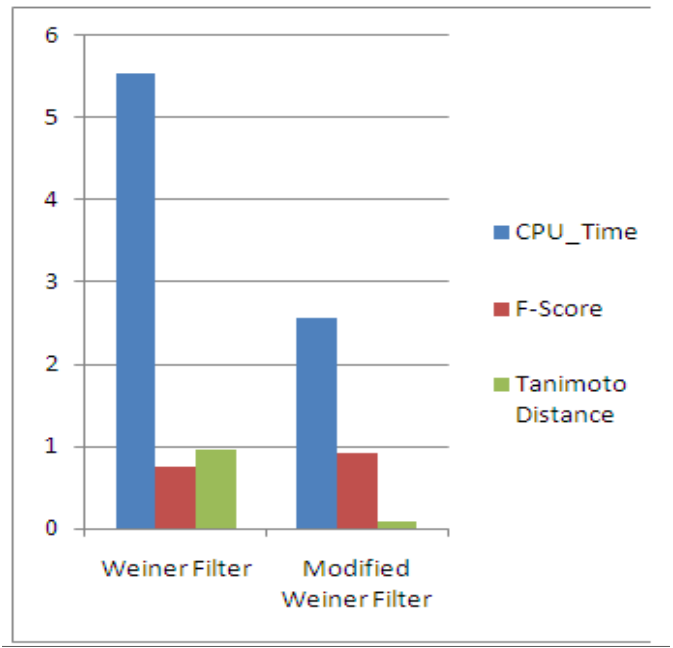

Figure 6: Comparison in terms of CPU_Time, F-Score and Tanimoto Distance

Result shows the variation as image is changed. However modified Weiner filter still shows better result as compared to existing Weiner filter.

Table 3: Comparison in terms of parameter values The plots of table 3 are shown as follows

\begin{tabular}{|l|l|l|l|}
\hline Image & Parameter & Weiner Filter & $\begin{array}{l}\text { Modified } \\
\text { Filter }\end{array}$ \\
\hline \multirow{2}{*}{ Image2 } & CPU Time & 5.9658 & 2.4568 \\
\cline { 2 - 4 } & F-Score & 0.8569 & 0.94576 \\
\hline & Tanimoto Distance & 0.95632 & 0.72562 \\
\hline
\end{tabular}




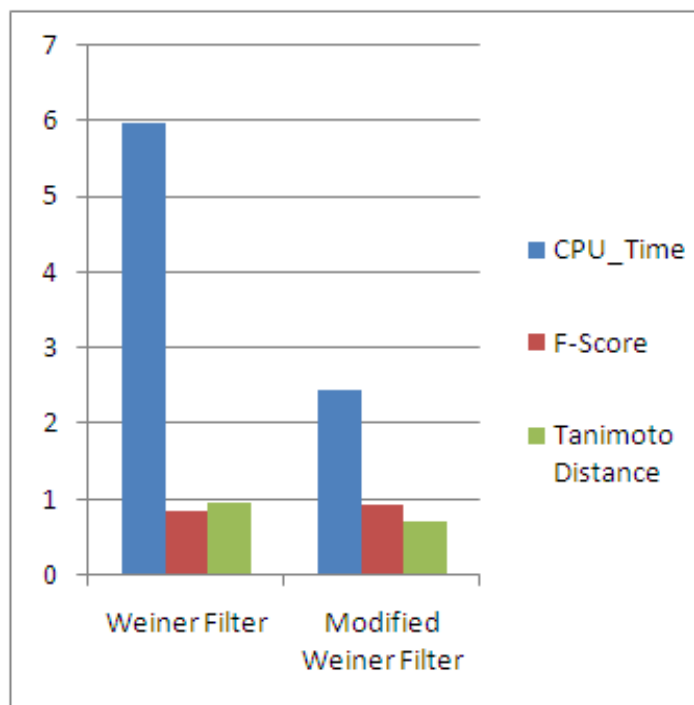

Figure 7: Result comparison in terms of image 2.

The comparison of results indicates the modified Weiner filter gives better result as compared to existing Weiner filter as used with MRI image.

\section{CONCLUSION AND FUTURE WORK}

The modified Weiner filtering when used for image restoration shows better result as compared to existing Weiner filter. The performance comparison proves the worth of proposed work. The dataset on which simulation is applied is taken from health care centers and internet. Clarity of image is desired in medical images. This study can help the medical professionals to diagnose the disease better as clarity is high. In addition of the clarity the size of the image is also reduced as overlapping pixels or pixels which are already plotted are eliminated.

In the future, the work can be extended towards coloured images also. The work which is proposed is limited to black and white MRI images only. So, enhancements in terms of image set are desired in the future.

\section{REFERENCES}

[1] T. M. Fields, "SVD-Based Technique for Interference Cancellation and Noise Reduction in NMR Measurement of,” 2016.
[2] T. Nadu and T. Nadu, "Detect Breast Cancer using Fuzzy C means Techniques in Wisconsin Prognostic Breast Cancer ( WPBC ) Data Sets,” vol. 2, no. 5, pp. 614-617, 2013.

[3] K. Vunckx, P. Suetens, and J. Nuyts, "Effect of overlapping projections on reconstruction image quality in multipinhole SPECT,” IEEE Trans. Med. Imaging, vol. 27, no. 7, pp. 972 983, 2008.

[4] V. G. Moruzzi, "AN INPAINTING TECHNIQUE BASED ON REGULARIZATION TO REMOVE BLEEDTHROUGH FROM ANCIENT DOCUMENTS I . Gerace , C . Palomba Dipartimento di Matematica e Informatica Universit `a degli Studi di Perugia A . Tonazzini Istituto di Scienza e Tecnologie dell ' Inf."

[5] “Wiener Filtering,” pp. 195-211, 2010.

[6] D. Regularization, R. H. Chan, C. Ho, and M. Nikolova, "Salt-and-Pepper Noise Removal by Median-type Noise Detectors and," pp. 1-14.

[7] E. J. Leavline, D. A. Antony, and G. Singh, "Salt and Pepper Noise Detection and Removal in Gray Scale ImagesAn Experimental Analysis,” vol. 6, no. 5, pp. 343-352, 2013.

[8] A. Kumari, P. J. Thomas, and S. K. Sahoo, "Single image fog removal using gamma transformation and median filtering," 11th IEEE India Conf. Emerg. Trends Innov. Technol. INDICON 2014, 2015.

[9] P. S. J. Sree, P. Kumar, R. Siddavatam, and R. Verma, "Saltand-pepper noise removal by adaptive median-based lifting filter using second-generation wavelets," Signal, Image Video Process., vol. 7, no. 1, pp. 111-118, Feb. 2011.

[10] R. H. Chan, C. W. Ho, and M. Nikolova, "Salt-and-pepper noise removal by median-type noise detectors and detailpreserving regularization,” IEEE Trans. Image Process., vol. 14, no. 10, pp. 1479-1485, 2005.

[11] S. V. Vaseghi, "Wiener Filters," Adv. Digit. Signal Process. Noise Reduct., vol. 9, pp. 178 - 204, 2000.

[12] N. Shimkin, “The Wiener Filter,” Estim. Identif. Dyn. Syst., no. 048825, pp. 1-15, 2009.

[13] A. Sri Krishna, G. Srinivasa Rao, and M. Sravya, "Contrast Enhancement Techniques Using Histogram Equalization Methods on Color Images With Poor Lightning," Int. J. Comput. Sci. Eng. Appl., vol. 3, no. 4, pp. 15-24, 2013.

[14] K. Gu, G. Zhai, S. Wang, M. Liu, J. Zhoi, and W. Lin, “A general histogram modification framework for efficient contrast enhancement," in 2015 IEEE International Symposium on Circuits and Systems (ISCAS), 2015, pp. 2816-2819.

[15] N. V. S. Malothu Nagu ${ }^{1}$, "Image De-Noising By Using Median Filter and Weiner Filtering," Int. J. Innov. Res. Comput. Commun. Eng., pp. 5641-5649, 2014. 